\title{
An unusual Erdheim-Chester disease with orbital involvement: a case report
}

\author{
Giuseppe Brunori, ${ }^{1}$ Aurelio Seidita, ${ }^{1}$ Lydia Giannitrapani, ${ }^{1}$ Antonietta Serruto, ${ }^{1}$ Francesco Bencivinni, ${ }^{2}$ \\ Maurizio Soresi ${ }^{1}$ \\ ${ }^{1}$ Biomedical Department of Internal Medicine; ${ }^{2}$ Centralized Imaging Diagnostics Service, University Hospital of Palermo, Italy
}

\begin{abstract}
Erdheim-Chester disease is a rare non-Langerhans cell histiocytosis with multiorgan involvement and a specific tropism for perivascular and fatty connective tissue, of unclear origin, with poor response to therapy. Its identification is difficult because of the variable clinical presentation and its lack of knowledge. We report the case of a 63-years-old woman, with a history of bilateral orbital pseudotumor, who comes to our attention because of progressively worsening asthenia, vomiting and systemic inflammation. Total body computerized tomography scan showed a volumetric increase of choroid plexus of the temporal horn of the left lateral ventricle, presence of solid retrobulbar tissue at the level of both maxillary sinuses, lung fibrosis, and retroperitoneal and peri-aortic infiltration. The association of these signs addressed to a diagnosis of Erdheim-Chester disease. Thus, although extremely rare, the diagnosis of Erdheim-Chester disease must be considered in the case of bilateral retro-orbital tumors and multisystemic involvement.
\end{abstract}

\section{Introduction}

Erdheim-Chester disease (ECD) is a rare nonLangerhans cell histiocytosis firstly described in $1930,{ }^{1}$ since then in no more than 500 cases. It has

Correspondence: Giuseppe Brunori, Biomedical Department of Internal Medicine (DiBiMIS), University Hospital of Palermo, via del Vespro 144, 90127 Palermo, Italy. Tel.: +39.91.6552834 - Fax: +39.91.6552896

E-mail: eko83m@libero.it

Key words: Erdheim-Chester disease; non-Langerhans cell histiocytosis; bilateral retro-orbital tumors; multisystemic involvement.

Acknowledgments: we wish to thank Ms. Carole Greenall for revising the text.

Contributions: $\mathrm{GB}, \mathrm{AS}, \mathrm{MS}$ were major contributors in writing the manuscript; LG, revised it critically; AS, reviewed ECD treatment; FB, provided the radiological assessment. All authors read and approved the final manuscript.

Conflict of interest: the authors declare no potential conflict of interest.

Received for publication: 1 December 2015.

Revision received: 10 June 2016

Accepted for publication: 16 June 2016.

This work is licensed under a Creative Commons Attribution NonCommercial 4.0 License (CC BY-NC 4.0).

CCopyright G. Brunori et al., 2017

Licensee PAGEPress, Italy

Italian Journal of Medicine 2017; 11:64-70

doi:10.4081/itjm.2016.700 multiple organ involvement characterized by fibrosclerotic and inflammatory feature, typically involving the orbits and the retro-orbital tissue in $25 \%$ of cases, mostly bilaterally, determining exophthalmos, retro-orbital pain, oculomotor paralysis and loss of vision. ${ }^{2}$ It is characterized by a progressive course although the recent discovery of a BRAF V600E mutation in more than half of the cases allowed implementing specific therapies thus changing prognosis. ${ }^{3,4}$

We present a case of ECD with previous history of pseudotumor cerebri with bilateral orbital involvement, where the evidence of a multisystem involvement on fibrotic-inflammatory basis led to the suspicion and then to the diagnosis of the disease.

Written informed consent was obtained from the patient for publication of this Case report and any accompanying images.

\section{Case Report}

A 63-years-old Caucasian woman was hospitalized in our unit for malaise, vomiting for about a week and signs of systemic inflammation. Her pathological history was characterized by a residual left hemiparesis due to poliomyelitis in childhood and an episode of ischemic stroke in the right ponto-mesencephalic site during a previous hospitalization when she was 54 . She was also diagnosed as suffering from chronic pansinusitis with orbital extension, binocular manifestation of retro-orbital pseudotumor and bilateral apical pulmonary fibrosis with traction bronchiectasis.

In 2013 she performed oculist consult for the worsening of the ophthalmological disease, which diag- 
nosed bulbar proptosis with conjunctival chemosis and widespread corneal opacity. She also underwent otolaryngologist and maxillo-facial surgery consult with biopsy of the maxillary sinus whose culture test was negative and histological examination proved: $\mathrm{Fi}$ brovascular tissue with inflammatory infiltrate characterized by lymphocytes, foamy histiocytes and congestive phenomena. Unfortunately she refused further diagnostic investigations. In addition she reported history of progressive immobilization and osteoarticular pain mostly in the legs.

On admission the patient was in poor general clinical condition, alert, lucid, partially cooperative and oriented in space and time, normal breathing, slowed speech, supine obliged, blind and with hearing loss. She complained of nausea, vomiting, and exacerbation of osteoarticular pain.

The physical examination showed pallor and dehydration of the skin and mucous membranes, bilateral bulbar proptosis with conjunctival chemosis and purulent secretions (Figure 1), muscle wasting in the lower limbs with complete ankylosis of the left knee joint and partial ankylosis of the right one. Blood tests showed neutrophilic leukocytosis, increased inflammatory markers, mild normochromic normocytic anemia, mild hyponatremia and hypoalbuminemia (Table 1). According to the medical history an esophagus-gastroduodenoscopy and a total body computerized tomography (CT) with contrast were performed to reassess the already known neurological and ocular pictures and to identify a possible occult thoracic or abdominal malignancy. Endoscopy proved negative, whereas CT showed an increased volume of choroid plexus of the temporal horn of the left lateral ventricle, presence of solid retrobulbar tissue at the level of both maxillary sinuses with diffuse bone rehash, apical pulmonary fibrosis associated with bronchiectasis, presence of solid tissue wrapping the left top bronchus and circumferential thickening of the walls of the infrarenal abdominal aorta (Figure 2).

A brain magnetic resonance (MR) was also performed with evidence of diffuse transcompartmental splanchnic and intracranial hypointensity, on T2W sequences, and enhancement after contrast, on $\mathrm{T} 1 \mathrm{~W}$, in particular the choroid plexus, the paranasal sinuses, the parapharyngeal structures and the left carotid space with significant pre-occlusion (Figure 3).

A total body positron-emission tomography (PET) further defined the multiorgan thoracic and abdominal involvement. Unfortunately, it was not possible to perform a long bone x-ray due to lack of cooperation of the patient who could not assume an appropriate posture for the investigation as a result of ankylosis of lower limb and osteoarticular pain during postural changes.

In the suspicion of a fibrotic histiocytic systemic disease, we asked for a revision of the previous maxillary sinus biopsy; our pathologists confirmed the already known inflammatory infiltrate but the immunohistochemical evaluation identified a specific CD68 positivity and CD1a negativity, compatible with the diagnosis of ECD.

During the hospitalization it was administered a systemic antibiotic therapy with quinolones and a local ocular aminoglycoside, low-dose steroid and analgesics with steady improvement in the local eye inflammatory picture and in the systemic inflammatory indexes. For the gastrointestinal symptoms levosulpiride and domperidone were administered with improvement of nausea and cessation of vomiting. Once the diagnosis was achieved, the patient was addressed to a reference center of immunology and rheumatology to undertake specific therapy and subsequent follow up.

After three months, we contacted the patient's relatives to know her conditions, but they reported a lack of will to pursue the path of follow-up and treatment suggested.

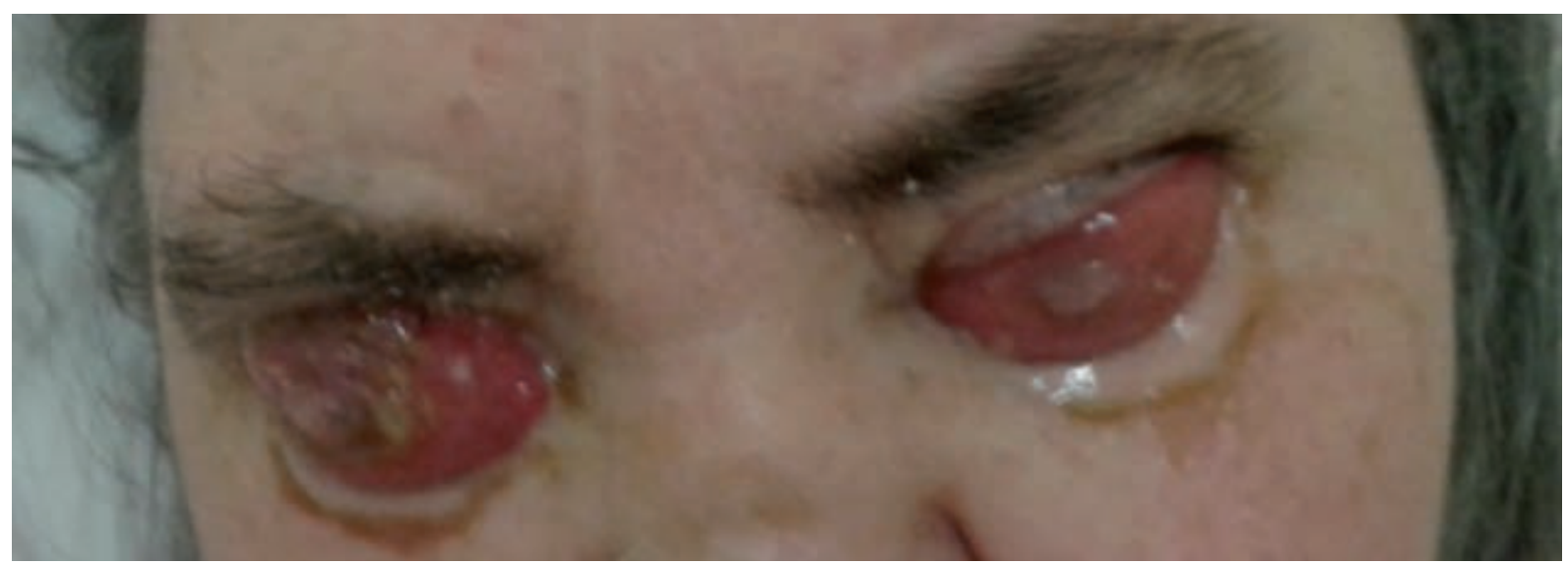

Figure 1. Bilateral bulbar proptosis with conjunctival chemosis and purulent secretions. 


\section{Discussion}

In our report, the multiorgan involvement and the specific tropism for perivascular and fatty connective tissue, together with the CD68+ and CD1a- histology, suggested the diagnosis of ECD. The rarity, the variable clinical presentation and the lack of knowledge about this disease make its identification a real challenge for physicians. ${ }^{1}$ Clinical and radiographic features can direct the diagnosis, but the clinical spectrum ranges from asymptomatic forms to clinical frameworks characterized by extensive infiltration and multisystem organ

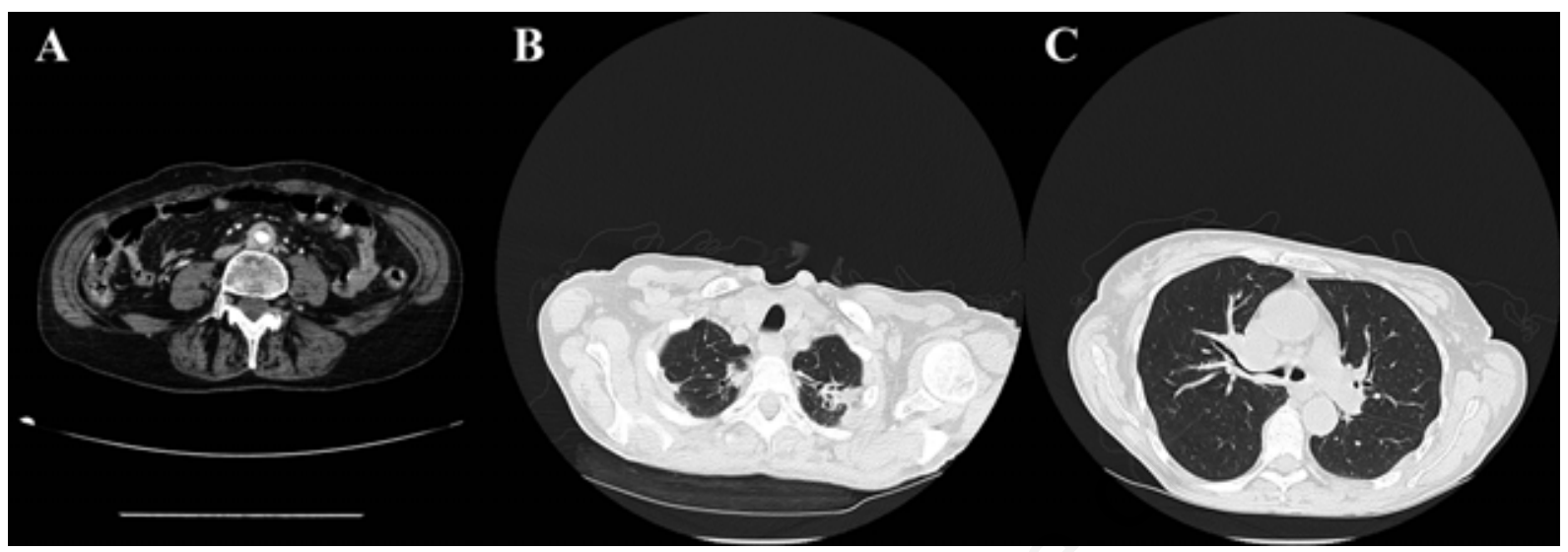

Figure 2. Enhanced abdomen computed tomography (CT) image showing the periaortic concentric soft tissue infiltration (A). Thoracic CT images showing apical lung fibrosis and bronchiectasis associated with the presence of solid tissue that like a sleeve wraps the left upper bronchus $(B, C)$.

Table 1. Laboratory parameters at admission and discharge.

\begin{tabular}{|c|c|c|c|c|}
\hline Laboratory tests & Entrance & Discharge & Unit & References values \\
\hline Erythrocytes & $3,780,000$ & $3,520,000$ & $10^{6} / \mu \mathrm{L}$ & $3.8-5$ \\
\hline Hemoglobin & 11 & 10.2 & $\mathrm{~g} / \mathrm{dL}$ & $12-16$ \\
\hline Hematocrit & 31.1 & 28.5 & $\%$ & $35-48$ \\
\hline $\mathrm{MCV}$ & 82.3 & 83.9 & $\mathrm{fL}$ & $80-99$ \\
\hline $\mathrm{MCH}$ & 29.1 & 29 & $\mathrm{pg}$ & $26-32$ \\
\hline Leukocytes & 17,910 & 10,260 & $10^{3} / \mu \mathrm{L}$ & $4-11$ \\
\hline Neutrophils & 65.3 & 58.8 & $\%$ & $40-74$ \\
\hline Lymphocytes & 26.8 & 28.8 & $\%$ & $20-48$ \\
\hline Monocytes & 6.9 & 4.8 & $\%$ & $3-11$ \\
\hline Eosinophils & 0.9 & 7.3 & $\%$ & $0-8$ \\
\hline Basophils & 0.1 & 0.3 & $\%$ & $0-1.5$ \\
\hline Platelets & 326,000 & 267,000 & $10^{3} / \mu \mathrm{L}$ & $150-450$ \\
\hline Azotemia & 29 & 10 & $\mathrm{mg} / \mathrm{dL}$ & $10-50$ \\
\hline Creatinine & 0.5 & 0.5 & $\mathrm{mg} / \mathrm{dL}$ & $0.51-0.95$ \\
\hline Sodium & 131 & 135 & $\mathrm{mEq} / \mathrm{L}$ & $136-145$ \\
\hline Potassium & 3.8 & 4.02 & $\mathrm{mEq} / \mathrm{L}$ & $3.3-5.1$ \\
\hline Calcium & 9.9 & 9.08 & $\mathrm{mg} / \mathrm{dL}$ & $8.4-10.2$ \\
\hline Sideremia & 31 & 52 & $\mu \mathrm{g} / \mathrm{dL}$ & $37-145$ \\
\hline Ferritin & 802 & 237 & $\mathrm{ng} / \mathrm{mL}$ & $15-150$ \\
\hline Transferrin & 144 & 195 & $\mathrm{mg} / \mathrm{dL}$ & $200-360$ \\
\hline CRP & 5.7 & 3.8 & $\mathrm{mg} / \mathrm{dL}$ & $0-0.5$ \\
\hline Fibrinogen & 580 & 402 & $\mathrm{mg} / \mathrm{dL}$ & $150-450$ \\
\hline Albumin & 3.1 & 3.0 & $\mathrm{mg} / \mathrm{dL}$ & $3.5-5.5$ \\
\hline
\end{tabular}

$\mathrm{MCV}$, mean corpuscular volume; $\mathrm{MCH}$, mean corpuscular hemoglobin; $\mathrm{CRP}, \mathrm{C}$-reactive protein. 
failure. $B R A F V 600 E$ mutation, an activating mutation of the proto-oncogene $B R A F$, has been recently identified in more than half of ECD patients, in accordance with a probable clonal origin of this disease. ${ }^{3}$ In parallel, in ECD patient compared to controls, it was found the expression of a proinflammatory cytokines pattern responsible for local activation and recruitment of histiocytes, with consequent elevated levels of interferon (IFN)- $\alpha$, interleukin (IL)-2, monocyte chemotactic protein- 1 and decreased IL-4 and IL-7. ${ }^{5}$

According to the most recent consensus guidelines $^{2}$ the diagnosis is based on:
- Histology: foamy or lipid- laden histiocytes with admixed or surrounding fibrosis. On immunohistochemical staining, histiocytes are positive for CD68, CD163, and Factor XIIIa, and negative for CD1a and Langerin (CD207). Positivity for S100 has been observed rarely. This differentiates ECD from Langerhans cell histiocytosis (LCH), where Langerhans cells are positive for CD1a, S100, and Langerin. ${ }^{2}$

- Skeletal abnormalities: symmetric diaphyseal and metaphyseal osteosclerosis of the long bones of the legs on X-ray or symmetric and abnormal intense labeling of the distal ends of the long bones of the

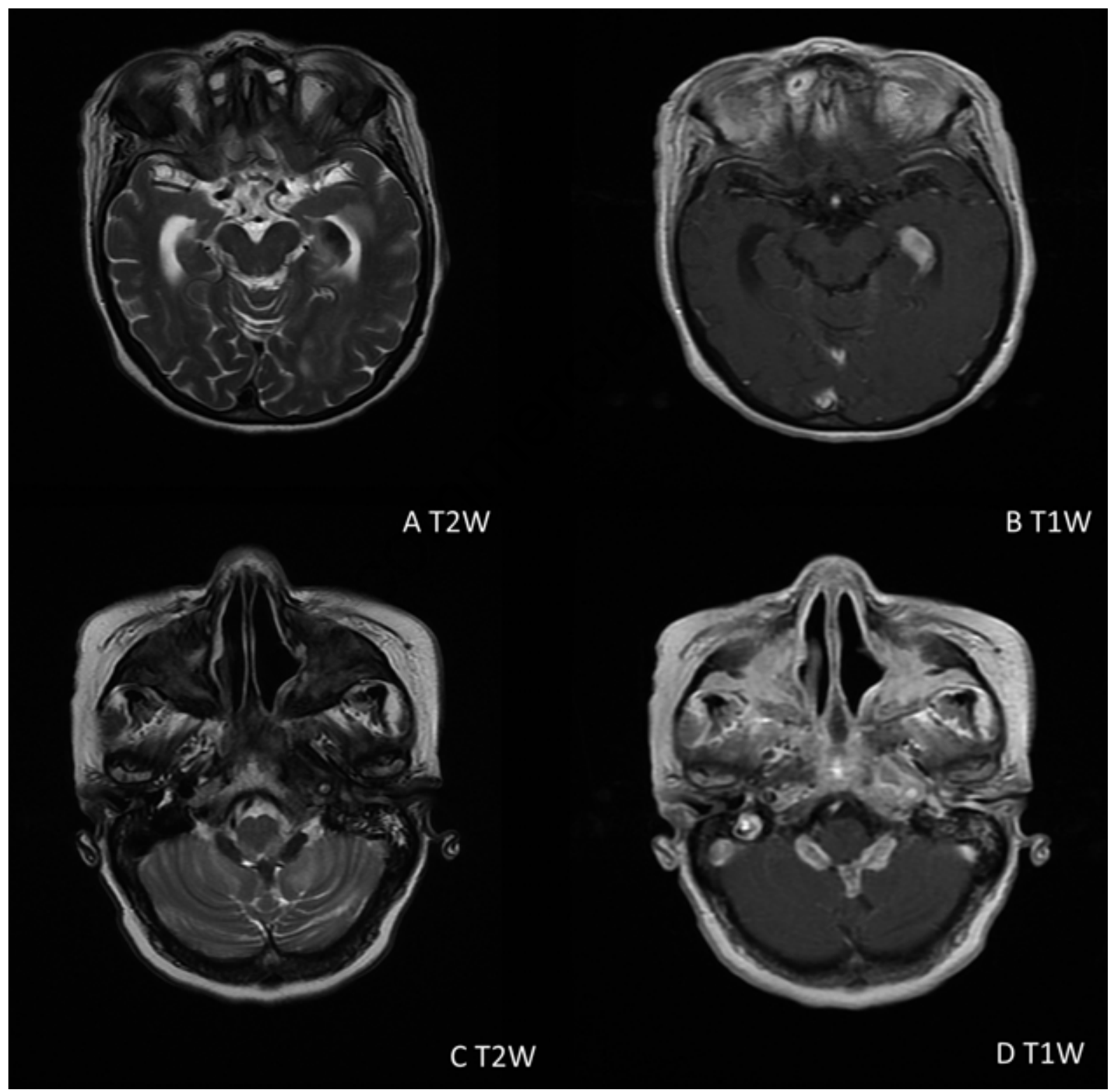

Figure 3. Axial T2 and T1 weighted with contrast magnetic resonance image. Hypointense tissue on T2 and enhancement after contrast on $\mathrm{T} 1$ in choroid plexus in the temporal horn of the left lateral ventricle involving orbital compartment bilaterally $(A, B)$, foramina of Luschka, terminations of the lower portion of the fourth ventricle and the maxillary sinuses bilaterally $(C, D)$. 
legs revealed by bone scintigraphy. ${ }^{2}$ Bone lesions may also be visualized less sensitively on CT, MR or PET imaging. ${ }^{6}$

Besides the skeletal involvement, present in more than $90 \%$ of patients, almost all organs are affected:

- Cardiovascular events: the most common manifestation is the circumferential involvement of thoracic and abdominal aorta with soft tissue called coated aorta. If renal artery is involved, it could develop renovascular hypertension. ${ }^{7}$ Pericardium may be involved in $40-45 \%$ of patients with pericarditis, pericardial effusion until tamponade. In one third of patients there can be infiltration pseudotumor of the right atrium. Cases of myocardial infarction by coronary involvement are described. ${ }^{7,8}$

- Pulmonary manifestations: In about half of the cases there is the involvement of lung and pleural parenchyma with thickening of interlobular septa, ground-glass opacities, centrilobular and pleural opacities, until the pulmonary fibrosis. ${ }^{9}$

- Nervous system and orbital manifestations: The nervous parenchyma may be diffusely infiltrated, so also hemispheric pachymeninx and cerebellar tentorium, as a meningioma. Then a generalized cognitive and motor function impairment can take place, symptoms of cerebellar nature ataxia and dysarthria, and in the case of orbits infiltration, exophthalmos, retro-orbital pain, oculomotor palsy and blindness. ${ }^{10}$

The infiltration of the orbits can be unilateral or bilateral, it occurs in $25 \%$ of patients and has to be distinguished from the ophthalmopathy during Graves' disease, granulomatous diseases, lymphoma and giant cell arteritis. ${ }^{11}$

- Endocrine manifestations: In 25\% of patients severe endocrinopathies occur including diabetes insipidus, hyperprolactinemia and hypopituitarism. ${ }^{12}$

- Retroperitoneal events: In 30\% of patients, it is possible to find striking images of retroperitoneal fibrosis and infiltration of the perirenal tissue with the typical feature of hairy kidney. ${ }^{2}$

- Skin manifestations: the most common is represented by xanthelasmas, yellowish maculopapular lesions on the face, neck, armpits and trunk present in one third of patients. ${ }^{2}$

The differential diagnosis is mainly with $\mathrm{LCH}$ and Rosai-Dorfman disease, with which it shares the infiltrative multisystem inflammatory involvement and the chronic-progressive trend but from which it differs for the peculiar skeletal involvement and the histopathology findings (Table 2 ). ${ }^{3}$

Other diseases that must be excluded are lymphomas, sarcoidosis, vasculitis, particularly Wegener's granulomatosis and Horton's arteritis. In all this cases the histology is diriment for diagnosis.

Unfortunately, to date, there are no curative thera- pies for ECD. The first-line treatment consists of IFN$\alpha$ (or pegylated-IFN). It was shown that IFN- $\alpha$ is the only treatment increasing patients survival, ${ }^{13}$ even if there are no randomized controlled trials on the efficacy of the drug. Treatment regimens ranges from $3,000,000$ to $9,000,000$ units three times a week depending on the level of organ involvement, therapeutic response and tolerability of the drug. ${ }^{13}$

Alternatively to IFN- $\alpha$, PEG-IFN is usually administered at doses of 135-200 $\mu$ g per week. ${ }^{13}$

IFN- $\alpha$ efficacy, however, is not the same on all the manifestations of the disease. Greater efficacy has been reported for the bone, skin, retro-orbital and pituitary localizations. ${ }^{14}$ Forms with cardiac, pulmonary and central nervous system involvement, which have the most unfavorable prognosis, ${ }^{9}, 13,14$ are less responsive and higher IFN- $\alpha$ doses may be necessary. Given the response variability as a consequence of organ involvement, for more severe forms alternative therapeutic approaches have been tested. One of the most promising is vemurafenib, a $B R A F$ inhibitor. The rational use of this drug is based on the pathogenic hypothesis of a clonal origin of the disease. In 54\% of patients with ECD was demonstrated the presence of BRAF V600E mutation at the level of histiocytes. ${ }^{3}$ Vemurafenib is effective in forms with severe multisystem involvement, refractory to IFN. ${ }^{4}$ Additional therapeutic alternative is represented by infliximab, a monoclonal anti tumor necrosis factor (TNF)- $\alpha$ antibody. TNF- $\alpha$ was, in fact, shown to play an important role in the regulation of the inflammatory pattern characterizing ECD. Infliximab has been proven especially in forms with cardiovascular involvement that less respond to IFN- $\alpha$. The treatment was effective, both in terms of symptoms and recovery of cardiac function. ${ }^{14,15}$ These treatments have been tried only on a limited number of patients and there are not yet available data on their efficacy and safety in the long term, for which further studies are required. Corticosteroids are traditionally used because of their anti-inflammatory action but have very limited effectiveness in ECD. Bisphosphonates may be employed for the treatment of bone lesions. On some disease manifestations, such as skin and bone lesions, a discrete effect has been reported for anakinra, IL-1 receptor antagonist. ${ }^{15}$

In our case, the patient was admitted because of gastrointestinal symptoms accompanied by signs of systemic inflammation, however, she showed clear signs of an infiltrative orbital and retro-orbital disease which had previously led to the diagnosis of retro-orbital pseudotumor.

Besides the neurological and orbital pictures to drive the suspicion of a multisystem disease there were the poor overall clinical conditions and the signs of systemic inflammation.

The execution of a deeper radiological investiga- 
tion and the evidence of the typical multisystem involvement allowed identification of a link between the initial neuro-ophthalmology diagnosis and the more complex picture, with fibrotic histiocytic genesis, finally diagnosed.

Helpful was also a previous retro-orbital tissue biopsy that already revealed the presence of the foam cells, typical of the ECD. According to the most recent diagnostic criteria, biopsy was re-examined by immunohistochemical analysis, proving compatible with ECD.

Peculiar was the retro-orbital and orbital involvement but also the extensive involvement of the neuro and splanchnocranium, especially choroid plexus, sinuses, para-pharyngeal structures and left carotid space with non-symptomatic pre-occlusion. The evidence of apical pulmonary fibrosis, extending to the left upper bronchus, wrapped sleeve by fibrotic tissue, the extensive involvement of the retroperitoneal space and the abdominal aorta testified an evolved disease condition. The previous clinical findings and diagnostic evaluations might have suggested a more detailed systemic evaluation in particular at the time of retroorbital pseudotumor and bilateral apical pulmonary fibrosis with traction bronchiectasis diagnosis.

It is important to focus on the clinical and radiological findings that may be bound to a fibrotic and inflammatory pathology with contemporary involvement of the brain, thoracic, abdominal and bone districts. The diagnosis of retro-orbital pseudotumor imposes more diagnostic tests in order to identify possible signs of multiple districts extension of the fibrotic process. A mention deserves gastrointestinal disorders that led the patient to the admission in our department. Esophagus-gastro-duodenoscopy excluded an organic genesis of vomiting, which resolved with symptomatic treatment and together with the resolution of local and systemic inflammatory state. The orbital and retro-orbital inflammatory state and the

Table 2. Systemic histiocytic disorders of adults. ${ }^{3}$

\begin{tabular}{|c|c|c|c|}
\hline & ECD & LCH & RDD \\
\hline \multicolumn{4}{|l|}{ Histopathological features } \\
\hline CD68 & 1 & 1 & 1 \\
\hline CD163 & 1 & 1 & 1 \\
\hline CD1a & 2 & 1 & 2 \\
\hline CD207 & 2 & 1 & 2 \\
\hline $\mathrm{S} 100$ & 2 or weakly & 1 & 1 \\
\hline Factor XIIIa & 1 & 2 & 2 \\
\hline Touton giant cells & 1 & 2 & 2 \\
\hline $\begin{array}{l}\text { Other characteristic features } \\
\text { of lesional histiocytes }\end{array}$ & $\begin{array}{c}\text { Xanthomatous features } \\
\text { Fibrosis }\end{array}$ & $\begin{array}{l}\text { Birbeck granules on electron } \\
\text { microscopy }\end{array}$ & $\begin{array}{l}\text { Intracytoplasmic lymphocytes } \\
\text { (emperipolesis) }\end{array}$ \\
\hline \multicolumn{4}{|l|}{ Organ system involvement } \\
\hline Skin & $\begin{array}{c}\text { Xanthelasma } \\
\text { Yellow or red-brown plaque }\end{array}$ & Scaly erythematous patches & Firm, indurated papules \\
\hline My & $\begin{array}{l}\text { Pericardial effusion } \\
\text { ocardial infiltration right atrial mass } \\
\text { eriaortic sheathing (coated aorta) }\end{array}$ & Reported, but uncharacteristic & Reported, but uncharacteristic \\
\hline Lungs & $\begin{array}{c}\text { Interlobular septal thickening, } \\
\text { ground-glass or centrilobular } \\
\text { opacities on CT }\end{array}$ & $\begin{array}{l}\text { Nodular and cystic changes } \\
\text { in upper and middle lobes }\end{array}$ & Reported, but uncharacteristic \\
\hline Retroperitoneum & Perinephric infiltration & Reported, but uncharacteristic & Reported, but uncharacteristic \\
\hline Liver and spleen & Rare & Uncommon but constitutes high-risk disease & Reported, but uncharacteristic \\
\hline Bone & $\begin{array}{l}\text { Femurs and tibia } \\
\text { Bone pain }\end{array}$ & $\begin{array}{l}\text { Craniofacial bones, proximal limbs, } \\
\text { pelvis, scapula }\end{array}$ & Reported, but uncharacteristic \\
\hline Lymph nodes & Reported, but uncharacteristic Ui & Uncommon but constitutes high-risk disease & Cervical, axillary, inguinal \\
\hline CNS & $\begin{array}{c}\text { Cerebellar or brain stem lesions } \\
\text { Dural-based lesions } \\
\text { Brain parenchymal lesions }\end{array}$ & $\begin{array}{c}\text { Cerebellar or brain stem lesions } \\
\text { Dural-based lesions } \\
\text { Brain parenchymal lesions } \\
\text { Non-infiltrative neuro-degeneration }\end{array}$ & Dural-based lesions \\
\hline
\end{tabular}

ECD, Erdheim-Chester disease; LCH, Langerhans cell histiocytosis; RDD, Rosai-Dorfman disease; CT, computerized tomography; CNS, central nervous system. 
bulbar and intracranial hypertension, can justify those gastric symptoms.

Antibiotic and steroid treatment solved the context of acute inflammation, however it was not possible to observe the subsequent evolution of the disease after discharge because of the suggested follow-up interruption at the rheumatology center of reference.

\section{Conclusions}

This case demonstrates that the diagnosis of ECD is not easy despite the presence of some clinical and radiological typical signs, not always recognized and traced to this rare disease.

The presence of a retro-orbital pseudotumor, especially if in combination with multifocal fibrosis, should lead to suspicion of this condition, then to the subsequent histological and immunohistochemical confirmation.

A prompt diagnosis and an adequate staging of the disease allow the implementation of a specific therapy with positive effects on prognosis, otherwise certainly unfavorable.

\section{References}

1. Chester W. Lipoidgranulomatose. Virchows Arch Pathol Anat 1930;279:561-602.

2. Diamond EL, Dagna L, Hyman DM, et al. Consensus guidelines for the diagnosis and clinical management of Erdheim-Chester disease. Blood 2014;124:483-92.

3. Haroche J, Charlotte F, Arnaud L, et al. High prevalence of BRAF V600E mutations in Erdheim-Chester disease but not in other non-Langerhans cell histiocytoses. Blood 2012;120:2700-3.

4. Haroche J, Cohen-Aubart F, Emile JF, et al. Dramatic efficacy of vemurafenib in both multisystemic and refractory Erdheim-Chester disease and Langerhans cell histiocytosis harboring the BRAF V600E mutation. Blood 2013;121:1495-500.
5. Arnaud L, Gorochov G, Charlotte F, et al. Systemic perturbation of cytokine and chemokine networks in Erdheim-Chester disease: a single-center series of 37 patients. Blood 2011;117:2783-90.

6. Dion E, Graef C, Miquel A, et al. Bone involvement in Erdheim-Chester disease: imaging findings including periostitis and partial epiphyseal involvement. Radiology 2006;238:632-9.

7. Haroche J, Amoura Z, Dion E, et al. Cardiovascular involvement, an overlooked feature of Erdheim-Chester disease: report of 6 new cases and a literature review. Medicine (Baltimore) 2004;83:371-92.

8. Gupta A, Kelly B, McGuigan JE. Erdheim-Chester disease with prominent pericardial involvement: clinical, radiologic, and histologic findings. Am J Med Sci 2002; 324:96-100.

9. Arnaud L, Pierre I, Beigelman-Aubry C, et al. Pulmonary involvement in Erdheim-Chester disease: a single-center study of thirty-four patients and a review of the literature. Arthritis Rheum 2010;62:3504-12.

10. Lachenal F, Cotton F, Desmurs-Clavel H, et al. Neurological manifestations and neuroradiological presentation of Erdheim-Chester disease: report of 6 cases and systematic review of the literature. J Neurol 2006;253: 1267-77.

11. Khamseh ME, Mollanai S, Hashemi F, et al. ErdheimChester syndrome, presenting as hypogonadotropic hypogonadism and diabetes insipidus. J Endocrinol Invest 2002;25:727-9.

12. Arnaud L, Hervier B, Néel A, et al. CNS involvement and treatment with interferon- $\alpha$ are independent prognostic factors in Erdheim-Chester disease: a multicenter survival analysis of 53 patients. Blood 2011;117:2778-82.

13. Hervier B, Arnaud L, Charlotte F, et al. Treatment of Erdheim-Chester disease with long-term high-dose interferon- $\alpha$. Semin Arthritis Rheum 2012;41:907-13.

14. Dagna L, Corti A, Langheim S, et al. Tumor necrosis factor $\alpha$ as a master regulator of inflammation in Erdheim-Chester disease: rationale for the treatment of patients with infliximab. J Clin Oncol 2012;30:e286-90.

15. Munoz J, Janku F, Cohen PR, Kurzrock R. ErdheimChester disease: characteristics and management. Mayo Clin Proc 2014;89:985-96. 